EPJ Web of Conferences 3, 01011 (2010)

DOI:10.1051/epjconf/20100301011

(C) Owned by the authors, published by EDP Sciences, 2010

\title{
Effective Field Theory and the No-Core Shell Model
}

\author{
I. Stetcu ${ }^{a}$ \\ Department of Physics, University of Washington, Seattle, WA, USA
}

\begin{abstract}
In finite model space suitable for many-body calculations via the no-core shell model (NCSM), I illustrate the direct application of the effective field theory (EFT) principles to solving the many-body Schrödinger equation. Two different avenues for fixing the low-energy constants naturally arising in an EFT approach are discussed. I review results for both nuclear and trapped atomic systems, using effective theories formally similar, albeit describing different underlying physics.
\end{abstract}

\section{Introduction}

Significant progress has been reported in the last decade toward achieving the holy grail of nuclear theory: a first principle description of the properties of atomic nuclei. This success is due to both a better understanding of the internucleon interactions, as well as the advent of increased computing power, supported by the development of sophisticated numerical algorithms.

The nuclear problem is pathologically complicated: on one hand, the inter-nucleon interaction is non-central, nonlocal and unconstrained at short distances, and on the other hand, the complexity of solving the many-body Schrödinger equation increases dramatically with the number of nucleons. Phenomenological and one-boson exchange models have provided guidance and have proved successful in applications to light nuclei; but a deeper understanding of the interactions between nucleons has been achieved using effective field theories (EFTs) [1-3], which provide interactions consistent with the symmetries of the underlying theory of the strong interactions, QCD. Moreover, EFT eliminates the model dependence (e.g., there are an infinite number of nucleon-nucleon interactions which are phaseshift equivalent), explains naturally the hierarchy of the nuclear interactions, and can mitigate shortcomings with the description of low-momentum observables, such as quadrupole transitions.

At the same time, several methods are now available for solving the nuclear few-body problem, such as Green's function Monte Carlo (GFMC) [4], Fadeev, Fadeev-Yakubovsky, (effective-interaction) Hyperspherical Harmonics $[5,6]$, and the no-core shell model (NCSM) [7,8], and they all agree within the statistical or numerical errors inherent to each method. Of these, the NCSM, while subject to greater errors, is the only one with the potential to be extended to heavier nuclei with fewer restrictions. ${ }^{1}$

\footnotetext{
a e-mail: istet@uw.edu

1 NCSM can handle both local and non-local interactions unlike GFMC that has difficulties in the presence of strong non-
}

In the conventional approach, the two issues have been considered decoupled: the effective interaction has been seen as merely an input to the many-body codes, with minimal mixing between the two worlds, required for the determination of the three-nucleon interaction parameters. In this paper, we review the efforts to combine the power of EFTs with the NCSM, with the goal of not only extending the benefits of QCD compatible solutions to a larger class of nuclei, but also of eliminating the model dependence and mitigating some shortcomings of the conventional NCSM. Reference [9] was a first attempt at generating effective interactions in NCSM restricted spaces, by fitting two- and three-body parameters directly to levels in light nuclei. An alternate method of fitting the two-body interaction was presented in Ref. [10], together with the first applications to few-atom systems in traps. We present briefly the two methods in Sec. 2, and applications to light nuclei and trapped atomic systems in Sec. 3, drawing conclusions and discussing further applications in Sec. 4.

\section{Theoretical framework}

\subsection{No-core shell model}

The NCSM is a few- and many-body technique specialized in solving the Schrödinger equation by direct diagonalization in a restricted basis set constructed with harmonic oscillator (HO) wave functions. The method has its roots in the phenomenological shell model, in which a residual effective interaction is diagonalized in a small model space, usually considering active only a small number of valence nucleons which are allowed to interact, while the remaining nucleons are frozen in a so called inert core. Whilst such an approach is quite successful in describing a large number of experimental data, the interaction is phenomenologically adjusted so that any connection with the underlying inter-nucleon interaction is completely lost.

localities, and is not restricted to (nearly) closed shells, as the coupled-cluster approach.

This is an Open Access article distributed under the terms of the Creative Commons Attribution-Noncommercial License 3.0, which permits unrestricted use, distribution, and reproduction in any noncommercial medium, provided the original work is properly cited. Article available at http://www.epj-conferences.org or http://dx.doi.org/10.1051/epjconf/20100301011 
The modern variant of the phenomenological shell model is the NCSM, where all the nucleons are allowed to interact. The non-relativistic intrinsic Hamiltonian describing such the system of $A$ protons and neutrons is

$$
H_{i n t}=\frac{1}{A} \sum_{i<j} \frac{\left(\mathbf{p}_{i}-\mathbf{p}_{j}\right)^{2}}{2 M_{N}}+\sum_{i>j=1}^{A} V_{i j}^{N N}+\sum_{i>j>k=1}^{A} V_{i j k}^{N N N}+\ldots
$$

where $V_{i j}^{N N}$ is the NN interaction, which depends only on the relative coordinate between the $i$ and $j$ particle, $V_{i j k}^{N N N}$ the NNN interaction, $\mathbf{p}_{i}$ the momentum of particle $i$, and $M_{N}$ the nucleon mass. Usually, $V^{N N}$ is fited with high accuracy low-energy observables (phase-shifts and deuteron properties), while the NNN interactions are adjusted to reproduce properties of the three-body system (tritium, nucleon-deuteron scattering). This Hamiltonian can be numerically diagonalized in an appropriate basis. There are two equivalent approaches to solving the many-body problem:

(i) relative (or Jacobi) coordinates: in this approach, the internal coordinates are described with $\mathrm{HO}$ wave functions [11]. Because in this method only relative coordinates are involved, the solutions are translationally invariant. Due to the complexity of the anti-symmetrization, this approach is efficient for nuclei up to $A=4$.

(ii) Slater determinant (SD) basis: from single $\mathrm{HO}$ basis states one constructs antisymmetric many-body basis states, SDs, in which the Hamiltonian is diagonalized. If one perform a truncation on the number of energy quanta above the non-interacting minimum configuration, one can separate exactly the center-of-mass (CM) motion, so that the results are free of spurious modes. While in this case the anti-symmetrization is trivial, the size of the many-body model space increases very quickly with the number of particles and the number of single-particle states. Even so, it remains the method of choice for $A>4$, and a lot of effort is dedicated toward the development of algorithms to handle larger and larger dimensions.

In principle, such an approach provides exact solutions to the Hamiltonian in Eq. (1). However, there are two issues that have to be addressed. First, the inter-nucleon interactions are not uniquely defined. Thus, as mentioned in the introduction, there are an infinite number of twobody interactions which describe the same set of observables (once one obtains a NN interaction, one can generate an infinite number of interactions via unitary transformations, which preserve the two-body observables). Second, for a complete calculation, an infinite number of $\mathrm{HO}$ states should be introduced in the calculations, and therefore the dimension of the many-body basis is infinite. Since the NCSM involves a numerical diagonalization, the manybody basis has to be truncated, and hence a method to include correlations left out by truncation are in order. In the NCSM, the truncation is determined by $N_{\max }$, which is the number of $\omega$ excitations above the minimum noninteracting configuration. Thus, the model space is defined by $N_{\max }$ and the frequency $\omega$. Note that the truncation in energy, given by $N_{\max }$ is related to the truncation in relative coordinates, so that calculations in relative coordinates and in a SD basis produce the same results for the same truncations. We concentrate in this section on the conventional solution to the second issue, and save the first for the next section.

In phenomenological NN interactions such as Argonne $v_{18}$ [12] the short-range part has been parametrized as a repulsive code of the order of $1 \mathrm{GeV}$. As a consequence, even low-lying states have considerable high-momentum components, making the convergence in finite model spaces very difficult. The unitary transformation approach takes into account the eliminated states to produce an effective interaction for the truncated model space. Any truncation induces up to $A$-body interactions, so an effective interaction that reproduces exactly the low-lying spectrum is as difficult to derive as solving the $A$-body problem. Hence, the so called cluster approximation has been derived, where the interaction is obtained for $a<A$ particles, and the result used in the $A$-body problem. In the following, we describe shortly the $a=2$ cluster approximation, as this approach also serves as motivation for the approaches proposed in Refs. $[9,10]$ and reviewed in Sec. 3.

Formally, one starts with the Hamiltonian in Eq. (1), but following Lipkin's idea [13], one adds the CM Hamiltonian, which does not modify the intrinsic properties of the nucleus, so that the $A$-body Hamiltonian reads

$$
\begin{aligned}
H_{A} & =H_{i n t}+\frac{\mathbf{P}_{C M}^{2}}{2 M_{N} A}+\frac{1}{2} A M_{N} \omega^{2} \mathbf{R}_{C M}^{2} \\
& =\sum_{i=1}^{A} h_{i}+\sum_{i>j=1}^{A}\left(V_{i j}-\frac{M_{N} \omega^{2}}{2 A}\left(\mathbf{r}_{i}-\mathbf{r}_{j}\right)^{2}\right) \\
& +\sum_{i>j>k=1}^{A} V_{i j k}^{N N N}+\ldots,
\end{aligned}
$$

where $h_{i}$ stands for a single-particle HO Hamiltonian of frequency $\omega$.

In the two-body cluster approximation, one considers the two-body problem

$$
h_{12}=h_{1}+h_{2}+V_{12}-\frac{M_{N} \omega^{2}}{2 A}\left(\mathbf{r}_{1}-\mathbf{r}_{2}\right)^{2}
$$

The two-body Hamiltonian $h_{12}$ is further separated into a relative contribution and a pair CM contribution, so that the previous equation can be cast as

$$
h_{12}=h_{r e l}^{A}+H_{12}^{C M},
$$

where

$$
h_{r e l}^{A}=\frac{p^{2}}{2 M_{N}}+\frac{1}{2} M_{N} \omega^{2}\left(1-\frac{2}{A}\right) r^{2}+V_{12} .
$$

and $\mathbf{p}=\left(\mathbf{p}_{1}-\mathbf{p}_{2}\right) / \sqrt{2}$ and $\mathbf{r}=\left(\mathbf{r}_{1}-\mathbf{r}_{2}\right) / \sqrt{2}$. The twobody correlations are then taking into account via a unitary transformation [14-17], which affects only the relative Hamiltonian $h_{r e l}^{A}$. Thus, the effective interaction is determined by the condition that the effective two-body 


\section{$19^{\text {th }}$ International IUPAP Conference on Few-Body Problems in Physics}

Hamiltonian $h_{\text {eff }}^{A}$ in the model space has the same lowlying eigenvalues as the original Hamiltonian $h_{r e l}^{A}$ in the full space. For further details and extension to an arbitrary $a$-body cluster we refer the interested reader to Ref. [7,8]. We just note that, by construction, in the limit in which the size of the model space goes to infinity, the starting Hamiltonian is recovered.

The NCSM approach, as briefly described here, has been applied successfully to the description of a large number of properties of low-lying states in light nuclei [7,18$22]$ and to predict properties relevant to the physics beyond the Standard Model [23]. However, the cluster approximation employed in these calculations is not justified a priori, and is was shown that treating low-momentum observables on the same footing with the Hamiltonian in the two-body cluster approximation is very challenging [24], motivating the search for a more flexible approach.

\subsection{EFTs}

The nucleons are not fundamental particles, and, in principle, the internucleon interactions are the direct consequences of the interactions between quarks and gluons inside nucleons. Such interactions are described by the QCD Lagrangian, but their consideration for the description of low-momentum observables of nuclei would be an overkill. EFTs provide a modern understanding of the theory of nuclear forces at low energies [25], even in the absence of exact solutions from QCD. In EFT, the interactions themselves are only defined in the context of a model space. Through the so-called low-energy constants (LECs) fixed to experimental data, the effective interactions contain information about the high-energy physics, whether known or unknown.

The main idea behind EFT is that truncation to a model space generates all the terms allowed by the symmetries of the underlying theory [1-3], in the nuclear case the QCD. There is an infinite number of such terms, but they can be organized as an expansion of the relevant momentum scale over the nucleon mass, $Q / M_{N}$. Such an organization, called power counting, allows for a consistent truncation of the relevant terms in the nuclear Hamiltonian. Thus, from the QCD Lagrangian, one obtains at low energies the most general Hamiltonian with the appropriate degrees of freedom and symmetries, so that interactions between nucleons consist of pion exchanges and contact terms, which subsume the short-range dynamics. In this approach, the leading order (LO) contains the physics necessary to generate the bound states, while the subleading orders should be small enough to be treated in perturbation theory.

In EFT, the model space is defined by a ultraviolet cutoff $\Lambda$, which is taken to have dimension of momentum. $\Lambda$ represents an arbitrary separation between the short-range dynamics included explicitly through virtual high momenta, and that included implicitly through the potential. The interaction itself depends on the ultraviolet cutoff in such a way that the observables are, in principle, $\Lambda$-independent.

In practical calculations, there are two sources of errors. The first type of errors is induced by the ultraviolet cutoff $\Lambda$; such errors can be made arbitrary small by increasing $\Lambda$. Thus, at large enough $\Lambda$ the errors are dominated by the physics excluded explicitly in the calculation, because of the truncation to a finite number of terms in the Hamiltonian. I will illustrate such a case in an example in Sec. 3.2.1, in which a leading order term cannot describe observables in the presence of a physical range parameter even in the limit $\Lambda \rightarrow \infty$. The advantage is that, if the power counting is correct, the dependence can be made arbitrary small by including more and more terms in the calculation, thus making the predictions improvable order by order.

\section{No-core shell model as effective theory}

In this section, I present the NCSM as an effective theory. Thus, because the truncation in a $\mathrm{HO}$ basis can be treated as any other truncation, one can construct an interaction in the NCSM truncated model spaces based on the EFT power counting. That is, the interaction preserves the form in each model space, and the overall coupling constants of different terms in the Hamitonian are fixed to reproduce some low-energy observables, such as energy levels.

Before proceeding with the applications, however, one must define the ultraviolet cutoff $\Lambda$ associated with the model space in which the solution is obtained. Thus, since $\left(N_{\max }+\right.$ $3 / 2) \omega$ is the maximum energy allowed, we define $\Lambda$ as the momentum associated with this energy in the relative coordinate of two particles:

$$
\Lambda=\sqrt{M_{N}\left(N_{\max }+3 / 2\right) \omega} .
$$

I will consider two applications: one to light nuclei, in which $\omega$ is just a parameter of the basis, and in that case its dependence in the results will be removed. In the second application presented in Sec. 3.2, $\omega$ is a physical trap, and it is more convenient to define the dimensionless cutoff

$$
\Lambda b=\sqrt{2 N_{\max }+3},
$$

where $b=1 / \sqrt{m \omega / 2}$ is the parameter length associated with a trap of frequency $\omega$, the nucleon mass $M_{N}$ being replaced by the mass $m$ of the trapped particles.

\subsection{Light nuclei}

I first consider the application to nuclear systems. For simplicity, I consider only very low energies, regime where the pions can be treated as short-range dynamics, and therefore not present explicitly in the Hamiltonian. In this pionless EFT, the LO contributions to the two-body interaction are two contact interactions acting in $S$-wave [26,27]. In addition, in the triton channel there is a three-body contact interaction, which prevents the three-body collapse, so that the Hamiltonian in Eq. (1) becomes

$H_{\text {int }}=\frac{1}{2 M_{N} A} \sum_{i, j}\left(\mathbf{p}_{i}-\mathbf{p}_{j}\right)^{2}$ 


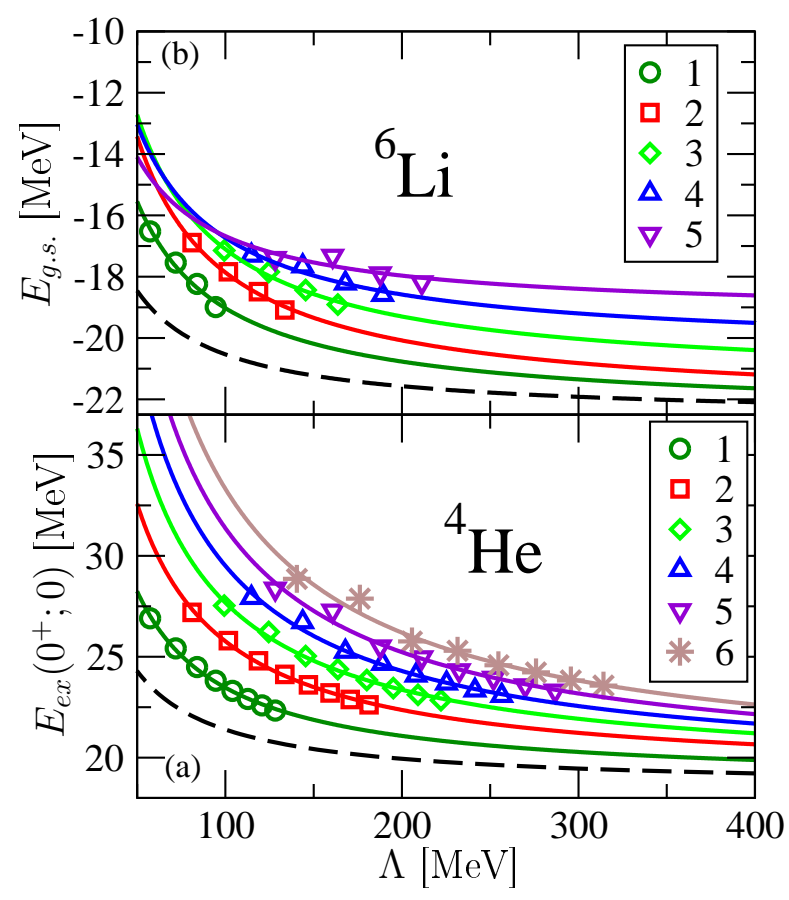

Fig. 1. Running with the ultraviolet cutoff for two observables: the first $\left(0^{+} ; 0\right)$ excited state in ${ }^{4} \mathrm{He}$ (lower panel) and the ${ }^{6} \mathrm{Li}$ ground-state energy (upper panel). The discrete points represent calculations for different frequencies, denoted in the legend in $\mathrm{MeV}$, while the continuous lines represent a fit to a linear dependence of $1 / \Lambda$ for fixed $\omega$. The dashed line represents the $\omega \rightarrow 0$ limit.

$$
\begin{aligned}
& +C_{0}^{0}\left(\omega, N_{\max }\right) \sum_{[i<j]^{0}} \delta\left(\mathbf{r}_{i}-\mathbf{r}_{j}\right)+C_{0}^{1}\left(\omega, N_{\max }\right) \sum_{[i<j]^{1}} \delta\left(\mathbf{r}_{i}-\mathbf{r}_{j}\right) \\
& +D_{0}\left(\omega, N_{\max }\right) \sum_{[i<j<k]} \delta\left(\mathbf{r}_{i}-\mathbf{r}_{j}\right) \delta\left(\mathbf{r}_{j}-\mathbf{r}_{k}\right)
\end{aligned}
$$

where $[i<j]^{S}$ denotes pairs of particles in the $S$-wave NN channel of spin $s$ and $[i<j<k]$ triplets of particles in the spin-1/2 $S$-wave $3 \mathrm{~N}$ channel. In this approach, the LECs depend on the model space, defined, as discussed in Sec. 2.1, by the HO frequency and the truncation $N_{\max }$. In a finite $\mathrm{HO}$ basis, only bound states can be accurately described. Therefore, while there are two LO couplings for the two-body contact terms, only $C_{0}^{1}\left(\omega, N_{\max }\right)$ can be fixed in each model space to the only NN bound system, the deuteron. The remaining constants, $C_{0}^{0}\left(\omega, N_{\text {max }}\right)$ and $D_{0}\left(\omega, N_{\max }\right)$ are adjusted in each model space, so that the tritium and the ${ }^{4} \mathrm{He}$ binding energies are reproduced. This is somehow different from the usual approach in EFT, where the two-body forces are fixed by the two-body scattering data.

With the coupling constants thus determined, one can solve the many-body problem, predict other states, and compare against the experimental data. Thus, in Fig. 1, the energy of the first $\left(J^{\pi} ; T\right)=\left(0^{+} ; 0\right)$ state in ${ }^{4} \mathrm{He}$ (lower panel) and the ground-state energy of ${ }^{6} \mathrm{Li}$ are plotted vs. the ultraviolet cutoff. Before discussing the results, it is useful to define, besides the ultraviolet cutoff, an infrared cutoff

$$
\lambda=\sqrt{M_{N} \omega},
$$

set by the spacing between HO levels. Note that because of the obvious connection between the infrared and ultraviolet cutoff, the latter cannot be increased by increasing the HO frequency $\omega$, as this would increase the errors associated with the infrared cutoff. Since, even for light nuclei, the calculations become quickly very demanding if one increases $N_{\max }$ too much, one has to consider an extrapolation $\Lambda \rightarrow \infty$, for a fixed infrared cutoff. Thus, manybody calculations are performed for a fixed frequency, denoted in Fig. 1 by the discrete symbols. Then, in order to increase the ultraviolet cutoff, we assume that the energy of the state runs linearly with the inverse of the ultraviolet cutoff for a fixed infrared cutoff. This particular running is motivated by the same type of running in the two-body sector [26,27], and is plotted in Fig. 1 with continuous lines. Finally, one takes the limit $\omega \rightarrow 0$ for a fixed $\Lambda$, which gives the dashed line in Fig. 1. Thus, the predicted first excited state in ${ }^{4} \mathrm{He}$ is within $10 \%$ of the experimental value, while the ${ }^{6} \mathrm{Li}$ binding energy is about $70 \%$ of experiment [9]. Although the LO results are not as precise as the ones obtained from phenomenological potentials, they are consistent with QCD, with the information about QCD contained in the parameters. Moreover, neglecting operators beyond the LO is expected to produce an error of about $30 \%$, so that the ${ }^{6} \mathrm{Li}$ result was considered the first successful application of the pionless EFT to $A>4$ nuclei. In principle, adding next-to-leading order terms and beyond improves the precision, as it happens in continuum calculations. Note that there is an extra source of errors: the assumption that for fixed $\omega$ the energy runs like $1 / \Lambda$. Thus adding an extra $\log \left(a_{0} \Lambda\right) / \Lambda$ term leaves the ${ }^{4} \mathrm{He}$ result virtually unchanged, but moves the result for the ground-state of ${ }^{6} \mathrm{Li}$ within $15 \%$ of the experimental result. However, more investigations are necessary in order to pin down the running with the ultraviolet cutoff. Finally, note that the excellent agreement for the first excited state in ${ }^{4} \mathrm{He}$ is not unexpected, as that state lies very close to the four-nucleon continuum threshold, well within the regime of applicability of the pionless EFT.

As a side note, it is interesting to find the limit of applicability of the pionless EFT for light nuclei, because, by construction, the theory breaks down if the processes described involve momentum transfer of order $m_{\pi}$ or greater. When we have a clear signal that it does fail, one can always apply the same principles to construct the interaction using the power counting in an EFT that includes pions (chiral EFT).

\subsection{Trapped systems}

In this section, I consider applications of the NCSM within a EFT framework to trapped few-body systems. This approach was introduced in Ref. [10], and was motivated by the search for a more convenient method to determine the LECs than the fit to bound states in light nuclei, as just discussed. Thus, the most convenient approach should involve fixing the LECs to the two-body data, i.e., phaseshifts. However, before I discuss the application to trapped 
$19^{\text {th }}$ International IUPAP Conference on Few-Body Problems in Physics

atomic systems, I will also argue about the relevance of such systems to the nuclear physics problem.

The experimental developments in the field of cold atoms have allowed one to reach a low-tunneling regime in three-dimensional optical lattices, so that each site is an essentially isolated harmonic trap occupied by few fermions $[28,29]$. Moreover, it is possible to tune the interaction between the constituents to a regime resembling the nuclear systems. Thus, the two-body scattering length $a_{2}$ can be made large with respect to the range of the interaction $r_{2}$, very similar to the case of two interacting nucleons at low energies.

The first approach to the problem of two particles interacting via short-range interactions, in the presence of the trap, was presented in Ref. [30], where the spectrum of the trapped system was derived under the assumption of particles interacting via a pseudopotential. The energy levels are thus given by the transcendental equation [30]

$$
\frac{\Gamma(3 / 4-\varepsilon / 2)}{\Gamma(1 / 4-\varepsilon / 2)}=\frac{b}{2 a_{2}},
$$

where $b=1 / \sqrt{\mu \omega}$ is the HO parameter length expressed in terms of the relative mass of two particles $(\mu=m / 2$ for equal mass particles) and $\varepsilon$ is the energy of the state in units of $\omega$. An EFT derivation of the Busch et. al. result will be given in a future publication [31]. This will allow a generalization $[32,33]$ to include all the parameters of the effective range expansion (ERE) as

$$
\frac{\Gamma(3 / 4-\varepsilon / 2)}{\Gamma(1 / 4-\varepsilon / 2)}=-\frac{1}{2} k b \cot \delta_{0}(k)
$$

with $k^{2}=2 \varepsilon / b^{2}$ the momentum associated with the energy $\varepsilon \omega$ and $\delta_{0}(k)$ is the $S$-wave phaseshift. Equation (10) and its generalization (11) provide the connection between the scattering observables (phaseshifts or low-energy parameters) and the energies of bound states of the trapped two-body system. Hence, because such states have boundstate asymptotics, they can be described in a finite $\mathrm{HO}$ basis. This approach overcomes the shortcoming of Sec. 3.1: if the particles are placed in a trap, Eqs. (10) or (11) provides the observables which can be used to fix LECs to the two-body system only. Thus, the low-lying states used for fit in the previous section could be either used to fit the three-body parameter, or can become predictions.

The question the reader might ask is why the trapped systems would be relevant for the nuclear system, where there is no physical trap. It turns out that, as hinted in Ref. [10], this approach was actually inspired from the conventional NCSM that involves the unitary transformation. Thus, while I have omitted in Sec. 2.1 a lot of details about the construction of such a unitary transformation, including the essential decoupling condition between the model and the excluded spaces, I have presented to an extensive length the procedure of adding the CM term. The main point is that adding a CM HO Hamiltonian produces a system that is formally trapped [13]. Furthermore, even in the conventional approach, the unitary transformation is not applied to a free space Hamiltonian, but to $h_{r e l}^{A}$ in Eq. (5), which contains a harmonic term. It is our intention to apply a similar procedure to the nuclear physics problem, with $V_{12}$ replaced by the contact interaction in Eq. (5), and the unitary transformation replaced by the power counting and the two-body renormalization described in the next section. However, before we attempt a first calculation in the nuclear system, we have tested this approach to the system of three two-component fermions at unitarity $\left(a_{2} \rightarrow \infty\right)$, for which a semi-analytical solution exists $[34,35]$.

\subsubsection{Two-body normalization}

In this section, I consider two spin- $1 / 2$ fermions in a harmonic trap described by the usual HO Hamiltonian:

$$
H_{0}=-\frac{\nabla^{2}}{2 \mu}+\frac{1}{2} \mu \omega^{2} r^{2}
$$

In LO, the potential between the two particles is a simple contact interaction acting in the $S$ state,

$$
V_{L O}(\mathbf{r})=C_{0} \delta(\mathbf{r})
$$

Thus, the Schrödinger equation describing the relative wave function $\psi(\mathbf{r})$ is

$$
\left(H_{0}+V_{L O}\right) \psi(\mathbf{r})=E \psi(\mathbf{r}) .
$$

As discussed before, any numerical diagonalization can be performed only in a finite model space. Formally, this reduces to the truncation of the wave function to the finite space. The natural basis to expand the wave function is $S$-wave eigenfunctions $\phi_{n}(\mathbf{r})=\phi_{n 00}(\mathbf{r})$ of the HO Hamiltonian (12). Thus, we write the relative wave function we include all basis states with principal quantum numbers up to $N_{\max }$ :

$$
\psi(\mathbf{r})=\sum_{n=0}^{N_{\max } / 2} c_{n} \phi_{n}(\mathbf{r})
$$

where $c_{n}$ are $N_{\max } / 2+1$ unknown coefficients to be determined from the diagonalization. At this point, besides the coefficients $c_{n}$, the coupling $C_{0}$ is also undetermined. In fact, at each value of $N_{\max }$ and $\omega, C_{0}\left(N_{\max }, \omega\right)$ is related to the eigenvalue $E$ [10]

$$
\frac{1}{C_{0}\left(N_{\max }, \omega\right)}=-\frac{\mu}{\pi^{3 / 2} b} \sum_{n=0}^{N_{\max } / 2} \frac{L_{n}^{(1 / 2)}(0)}{(2 n+3 / 2)-E / \omega} .
$$

Hence, we can fix the value $C_{0}\left(N_{\max }, \omega\right)$, so that the energy of one state reproduces exactly the values given by Eq. (10), or, more generally, Eq. (11). The natural choice is to use the ground state energy in the fit. In particular, at unitarity, if we choose to fix the ground state energy $\varepsilon_{0}=E_{0} / \omega=1 / 2$, the summation over $n$ can be performed analytically [36]:

$$
C_{0}\left(N_{\max }, \omega\right)=-\frac{\pi^{2} b}{2 \mu} \frac{\Gamma\left(\frac{1}{2} N_{\max }+1\right)}{\Gamma\left(\frac{1}{2} N_{\max }+\frac{3}{2}\right)},
$$


so that in the limit $N_{\max } \rightarrow \infty$

$$
\frac{\mu C_{0}\left(N_{\max }, \omega\right) \Lambda}{2 \pi} \rightarrow-\frac{\pi}{2}
$$

like in the continuum basis [27]. While the summation cannot be performed analytically for other values of $b / a_{2}$, the same running of the coupling is found numerically at large $N_{\text {max }}$ for arbitrary values of $b / a_{2}$ as shown in Fig. 1 of Ref. [10]. A detailed investigation of the running of the coupling constants will be presented in Ref. [31].

After we determine $C_{0}$ in a model space defined by $N_{\text {max }}$, one can solve numerically Eq. (14). Even if we consider a case with all the ERE parameters zero, except for the scattering length, solving Eq. (14) will yield a spectrum that deviates from the spectrum given by (10). Thus, the truncation introduces errors. In fact, even if the underlying physics would be described by a pseudopotential, which is the assumption in deriving Eq. (10), the truncation introduces an effective range, shape parameter, and so on. In Fig. 2, I present with dotted line the running of the first four excited states at unitarity, once the ground state is fixed to the ground state using Eq. (17).

As illustrated in Fig. 2 the errors decrease with increasing the model space. This is not the only way one can reduce the errors. Thus, in smaller model spaces, the errors can be reduced by adding more terms to the potential. For example, in NLO one can add a term

$$
V_{N L O}\left(\mathbf{r}, \mathbf{r}^{\prime}\right)=-C_{2}\left\{\left[\nabla^{\prime 2} \delta\left(\mathbf{r}^{\prime}\right)\right] \delta(\mathbf{r})+\delta\left(\mathbf{r}^{\prime}\right)\left[\nabla^{2} \delta(\mathbf{r})\right]\right\}
$$

in perturbation theory to correct the range to the physical value. Note that even if there is no physical range, the coefficient $C_{2}$ will not vanish in finite model spaces because the truncation induces by itself a range.

In general, the term in Eq. (18) will change the energy of the state used to fix the LO term. One can, however, fit $C_{0}$ and $C_{2}$ to reproduce at the same time two energies given by Eq. (10). This procedure can be simplified if one assumes that the change in $C_{0}$ is small and can be treated in perturbation theory, so that the full NLO potential writes

$$
\begin{aligned}
& V_{N L O}\left(\mathbf{r}, \mathbf{r}^{\prime}\right)=C_{0}^{(1)} \delta(\mathbf{r}) \delta\left(\mathbf{r}^{\prime}\right) \\
& \quad-C_{2}\left\{\left[\nabla^{\prime 2} \delta\left(\mathbf{r}^{\prime}\right)\right] \delta(\mathbf{r})+\delta\left(\mathbf{r}^{\prime}\right)\left[\nabla^{2} \delta(\mathbf{r})\right]\right\} .
\end{aligned}
$$

By diagonalizing the LO Hamiltonian (14), one obtains all the eigenvalues $\varepsilon_{i}^{L O}$ and eigenvectors $\psi_{i}^{L O}(\mathbf{r})$ in the finite model space. Therefore, one can calculate in first order perturbation theory corrections to the energies as expectation values:

$$
\Delta \varepsilon_{i}=\left\langle\psi_{i}^{L O}\left|V_{N L O}\right| \psi_{i}^{L O}\right\rangle .
$$

Assuming that the value of the ground-state energy $\varepsilon_{0}$ was fixed to the value given by Eq. (10), one equation that determines the coupling constants $C_{0}^{(1)}$ and $C_{2}$ is given by the condition that $\varepsilon_{0}$ does not changed from LO, i.e.,

$$
\Delta \varepsilon_{0}=0 .
$$

The second equation is obtained by requiring that the energy of a second level, e.g., the first excited level, reproduce the appropriate value given by Eq. (10) denoted by $\varepsilon_{1}^{e x}$

$$
\Delta \varepsilon_{1}=\varepsilon_{1}^{e x}-\varepsilon_{1}^{L O}
$$

An ever more involved, although strait forward procedure, which include a second-order perturbation theory correction of the $V_{N L O}\left(\mathbf{r}, \mathbf{r}^{\prime}\right)$ contribution, can be devised for the $\mathrm{N}^{2} \mathrm{LO}$ correction. More details will be given in a future publication [31].

As an illustration, I consider first the system of two trapped particles at unitarity $\left(b / a_{2}=0\right)$, with vanishing range $\left(r_{2} / b=0\right)$. The running with the dimensionless cutoff $b \Lambda$ of the energies of the first four excited states is shown in Fig. 2. The coupling constant $C_{0}$ is fixed from Eq. (17), to reproduce the lowest level given by (10), while the LO, NLO, and $\mathrm{N}^{2} \mathrm{LO}$ coupling constants are fixed at each order to reproduce $\varepsilon_{1}$ (at NLO and $\mathrm{N}^{2} \mathrm{LO}$ ), and $\varepsilon_{3}$ (at $\mathrm{N}^{2} \mathrm{LO}$ given by the same Eq. (10). As shown in Fig. 2, at large $b \Lambda$, the errors associated with the cutoff vanish. At fixed cutoff, corrections beyond LO improve the description of observables systematically, as expected.

Another case, of interest not only for nuclear physics but also for cold atoms, is the presence of a non-neglijible range. Thus, in the following example, I assume the two particles at unitarity, but set $r_{2} / b=0.15$ in Eq. (11), which determines the exact spectrum. The coupling constants are now adjusted to reproduce $\varepsilon_{0}$ (at LO, NLO, and $\mathrm{N}^{2} \mathrm{LO}$ ), $\varepsilon_{1}$ (at $\mathrm{NLO}$ and $\mathrm{N}^{2} \mathrm{LO}$ ) and $\varepsilon_{2}$ (at $\mathrm{N}^{2} \mathrm{LO}$ ) given by Eq. (11). As shown in Fig. 3, this case presents a significant difference with respect to the case without the range: except for $\varepsilon_{0}$, which is fixed by construction and not shown in the figure, the other levels are not reproduced at LO, even if one increases the cutoff. Thus, at large enough values of $b \Lambda$, the errors are dominated by the missing physics (the range term), rather than the cutoff induced errors. However, once the NLO term, containing the physics associated with the range, is added, the results improve systematically. Thus, the only errors in the description of the observables are associated with the cutoff, and they can be decreased by increasing the cutoff, or by introducing $\mathrm{N}^{2} \mathrm{LO}$ corrections. Finally, note that the procedure at LO, I have used the ground-state given by Eq. (11), even though there is no range term in the expansion of the potential. In continuum, this procedure is similar to fitting $C_{0}$ to reproduce the deuteron binding energy in the ${ }^{3} \mathrm{~S}_{1} \mathrm{NN}$ channel. An alternate procedure would involve using $\varepsilon_{0}$ given by Eq. (10) for fixing $C_{0}$, which is similar to fitting to the scattering length in the continuum EFT. Once the NLO term is added, the observables not already fixed approach the exact results, although the details of the running are different in the two cases.

\subsubsection{Three-body problem}

Once the two-body interaction is determined as described in the previous section, one can investigate systems with more particles. I consider in this paper only the case of three trapped fermions of spin $1 / 2$. One can show that the intrinsic energy spectrum of three spin- $1 / 2$ fermions in a 
$19^{\text {th }}$ International IUPAP Conference on Few-Body Problems in Physics
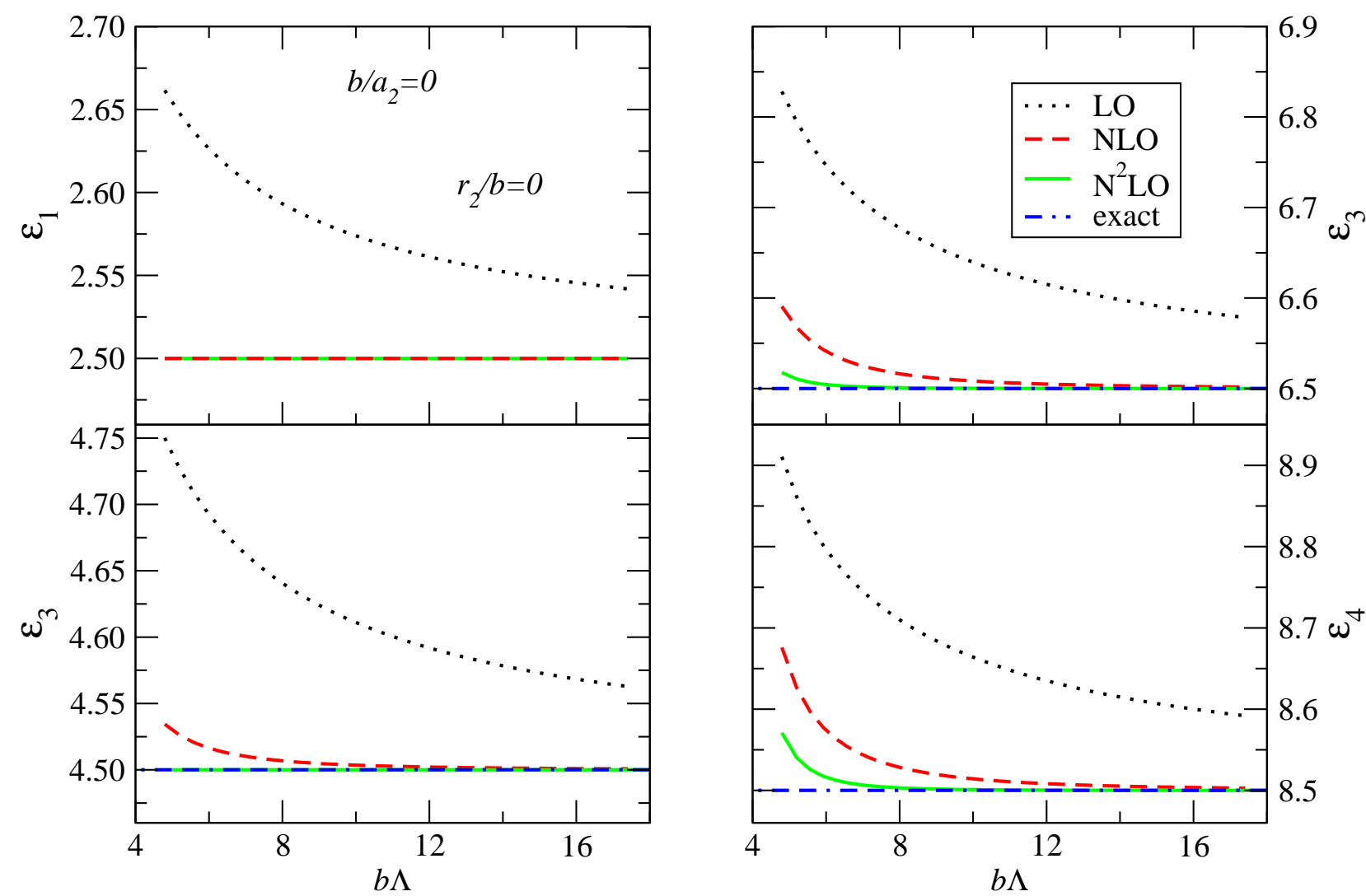

Fig. 2. Running of the energies of the first four excited states for two trapped particles at unitarity. The ground state is fixed in LO by means of Eq. (17). At NLO, the first excited state is used to fix $C_{2}$, in addition to the ground-state energy, while at $\mathrm{N}^{2} \mathrm{LO}$ the second excited state is also used to fix $C_{4}$. I assumed no physical effective range.

trap of frequency $\omega$, is given by $[34,35]$

$$
E_{3}=(1+s+2 q) \omega
$$

where $q \geq 0$ is an integer, while $s$ is a real positive number solution to a transcendental equation. According to the EFT principles, any truncation induces not only higher order two-body interactions (all allowed by the symmetries), but also many-body forces. However, for two-component fermions, three-body forces appear only at $\mathrm{N}^{3} \mathrm{LO}$ [37-41], so that the properties of three-body system are determined to a large degree by the two-body interactions alone.

As in the case of the two-body system, I iterate the LO to all orders, but treat the subleading orders in perturbation theory. Figures 4 and 5 illustrate the running of the lowest $L=1$ (upper panel) and $L=0$ (lower panel) energies of the trapped three-body system at unitarity. The two-body coupling constants for the two-body interactions were fixed to two-body observables, as described in the previous section, while the numerical three-body results were obtained with a code in relative coordinates [11], modified to handle both fermions and bosons of arbitrary spin.

In Fig. 4, I show the results for a system of three particles, assuming contact two-body interactions in $S$ waves with neglijible effective range. Because the range of cutoffs the three-body can handle is very limited, I have chosen to show the running with $N_{\max }$ rather than $b \Lambda$. As shown in Fig. 4, at large cutoffs, the three-body energies converge to the exact results (dot-dashed lines), as expected. Moreover, as advertised, the errors are reduced when the corrections from subleading order are added. Thus, while for the $L=1$ state (the ground state) the NLO energy appears to converge faster at low $N_{\max }$, it eventually overshuts the Werner and Castin result and converges from above. For the $L=0$ state, adding the NLO correction describes already with good accuracy the exact result.

The method presented here is general enough to be applied to arbitrary $b / a_{2}$ ratios, as well as non-zero ERE parameters. LO predictions for selected $b / a_{2}$ ratios have been presented in Ref. [10]. In particular, it is interesting to investigate the behavior of the lowest state of the three-body system in the limit of $b / a_{2} \rightarrow \infty$, i.e., the continuum limit. Thus, while at unitarity the three-body ground state has $L=1$, around $b / a_{2} \approx 1.5$, the lowest state has $L=0$ angular momentum. Moreover, in the limit $b / a_{2} \rightarrow \infty$, one can show that the energy of the three-body state is

$$
E_{3}=-\frac{1}{2 \mu a_{2}^{2}},
$$

which is the energy of a bound state of two particles.

Here, I present just another example, with three particles at unitarity, but with non-zero range, $r_{2} / b=0.20$. In Fig. 5, however, the LO is obtained in the absence of the range, so that is unchanged from Fig. 4. Thus, in this case, the two-body coupling is determined from Eq. (17), 

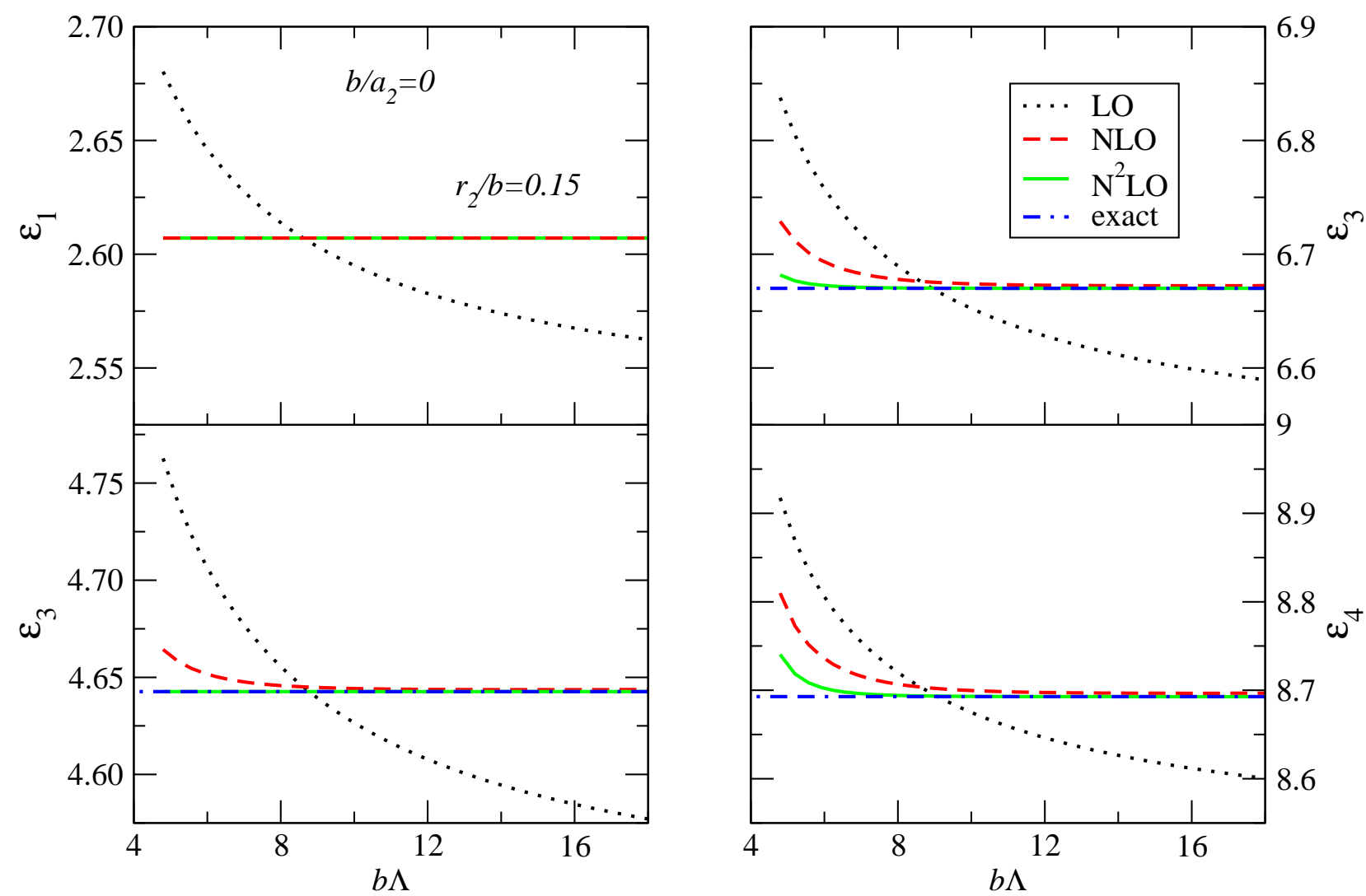

Fig. 3. Running of the energies of the first four excited states for two-trapped particles at unitarity with a non-vanishing physical range, $b / a_{2}=0.15$.The coupling constants are fixed so that the ground-state, and the first and second excited states given by Eq. (11) are fixed in the appropriate order: at $\mathrm{LO}, \varepsilon_{0}$, at $\mathrm{NLO}, \varepsilon_{0}$ and $\varepsilon_{1}$, and at $\mathrm{N}^{2} \mathrm{LO}, \varepsilon_{0}, \varepsilon_{1}$ and $\varepsilon_{2}$ respectively.

while the range corrections are added in subleading orders. Hence, one can study the effect of the range corrections to the three-body levels: while the $L=1$ is pushed up by about $1.5 \%$, the $L=0$ is pushed up by about $7 \%$ with respect to the results of Werner and Castin. More details and examples will be presented in a future publication [42].

\section{Conclusions and outlook}

In this contribution, I have presented a review of a direct application of EFT principles to the construction of effective interactions tailored to finite NCSM model spaces. In such applications, the ultraviolet cutoff is defined in terms of $N_{\max }$, the number of shells included in the NCSM calculation, and the frequency $\omega$ of the HO states used to construct the many-body states.

Two alternative procedures of fixing the LECs have been illustrated. As a first application, I have considered the nuclear system in a pionless EFT approach. In this case, there are three LECs, two coupling constants in the NN sector and one three-body parameter in the triton channel. The strength of the contact interaction in the ${ }^{3} S_{1}$ channel has been fixed to reproduce the deuteron binding energy in all the model spaces. Because the deuteron is the only two-body bound state, the strength of the two-body contact term in the ${ }^{1} \mathrm{~S}_{0}$ and the three-body parameters have been determined so that one simultaneously reproduces the triton and ${ }^{4} \mathrm{He}$ binding energies. Following an additional procedure of removing the infrared cutoff $\omega$ artifact, inherent to the $\mathrm{HO}$ basis, the energy of the first $\left(0^{+} ; 0\right)$ state in the alpha particle has been determined in very good agreement with the experiment. The ground-state energy of ${ }^{6} \mathrm{Li}$ is predicted with less accuracy [9], but still within 30\% of the experimental value. It is important to stress out that while the calculations have been carried out in a NCSM formalism and using an effective theory without pions, the same general principles can be applied to other many-body techniques, as well as to the chiral EFT.

The lack of a larger number of bound states in the NN system makes the application of this procedure difficult for subleading orders, or for the EFT with explicit pions. This has motivated the search for a more flexible procedure, in which all the two-body parameters are fixed in the two-body system alone. The harmonically trapped systems present the advantage that all the states are bound, and the natural basis states are the $\mathrm{HO}$ wave functions used in NCSM calculations. Moreover, it has been shown that the spectrum of two particles trapped in a HO potential is entirely determined by the ERE parameters in the continuum $[30,32,33]$. The energy levels in the trapped two-body system can be used to determine the LECs in a finite oscillator basis [10]. In this approach, we have applied the same procedure as in continuum: the physics at the LO was iterated 
$19^{\text {th }}$ International IUPAP Conference on Few-Body Problems in Physics

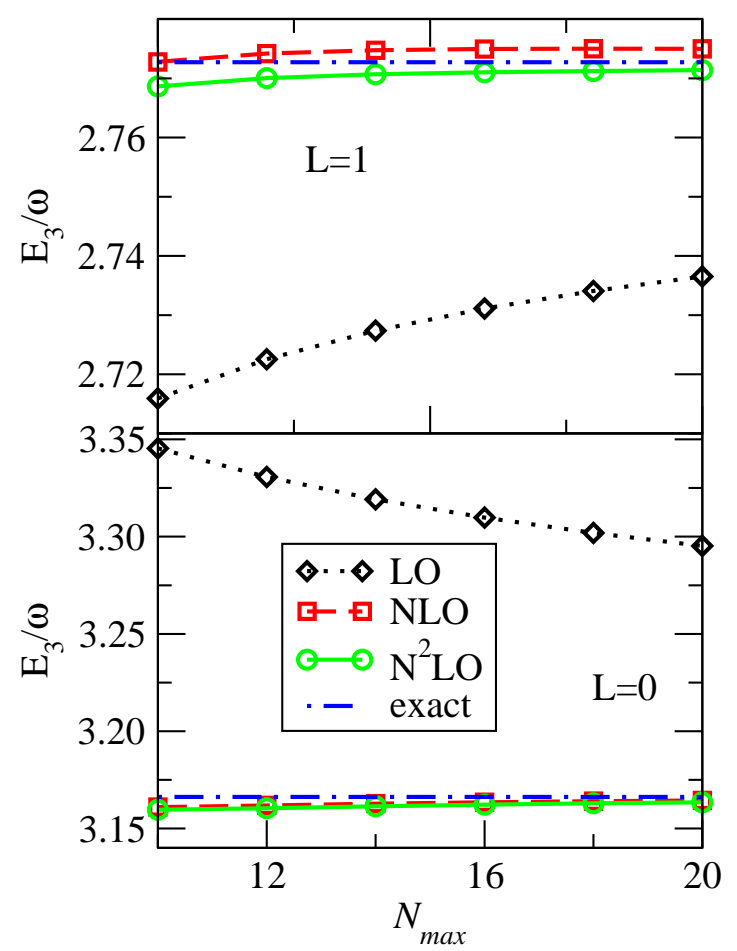

Fig. 4. The running of the lowest $L=0$ (lower panel) and $L=1$ levels at LO, NLO and $\mathrm{N}^{2} \mathrm{LO}$. I have considered two-body interactions at unitarity $\left(b / a_{2}=0\right)$ with no range $\left(r_{2} / b=0\right)$.

in all orders, while the subleading orders were treated in perturbation theory. Furthermore, application to the threebody system has shown that the exact solution at unitarity $[34,35]$ can be reproduced: the errors associated with the truncation can be eliminated either by increasing the cutoff [10], or by adding corrections beyond the LO [42]. Finally, the procedure can be easily extended to arbitrary $b / a_{2}$ ratios, as well as small values of $r_{2} / b$ ratios or other small ERE parameters. Obviously, this extension is of great interest for applications to the nuclear systems.

Because the system of cold atoms in the presence of Feshbach resonances presents remarkable similarities with the system of interacting nucleons at low energies, one can hope that the same procedures can be extended to the nuclear many-body problem. Work in this direction is in progress.

\section{Acknowledgments}

I thank my collaborators B. R. Barrett, M. Birse, J. Rotureau, and U. van Kolck for their contribution to the project. I also thank the organizers of the conference for giving me the opportunity to present the current work, as well as for the impeccable organization of the meeting. This work was supported by the UNEDF SciDAC Collaboration under DOE grant DE-FC02-07ER41457.

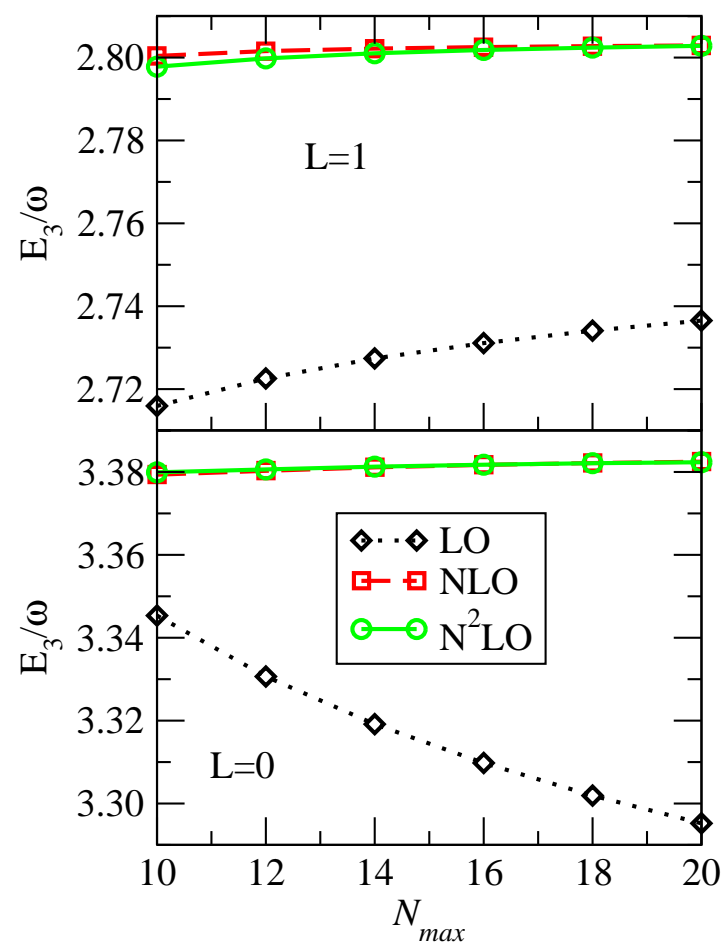

Fig. 5. Same as in Fig. 4, but for $r_{2} / b=0.20$.

\section{References}

1. S. Weinberg, Phys. Lett. B251, 288 (1990)

2. S. Weinberg, Nucl. Phys. B363, 3 (1991)

3. C. Ordóñez, U. van Kolck, Phys. Lett. B291, 459 (1992)

4. J. Carlson, Phys. Rev. C 36, 2026 (1986)

5. A. Kievsky, S. Rosati, M. Viviani, Nucl. Phys. A551, 241 (1993)

6. N. Barnea, W. Leidemann, G. Orlandini, Phys. Rev. C 61, 054001 (2000)

7. P. Navrátil, J.P. Vary, B.R. Barrett, Phys. Rev. C 62, 054311 (2000)

8. P. Navratil, S. Quaglioni, I. Stetcu, B.R. Barrett, J. Phys. G36, 083101 (2009)

9. I. Stetcu, B.R. Barrett, U. van Kolck, Phys. Lett. B653, 358 (2007)

10. I. Stetcu, B.R. Barrett, U. van Kolck, J.P. Vary, Phys. Rev. A 76, 063613 (2007)

11. P. Navrátil, G.P. Kamuntavicius, B.R. Barrett, Phys. Rev. C 61, 044001 (2000)

12. R.B. Wiringa, V.G.J. Stocks, R. Schiavilla, Phys. Rev. C 51, 38 (1995)

13. H.J. Lipkin, Phys. Rev. 109, 2071 (1958)

14. S. Okubo, Prog. Theor. Phys. 12, 603 (1954)

15. J. Da Providencia, C.M. Shakin, Ann. of Phys. 30, 95 (1964)

16. K. Suzuki, Prog. Theor. Phys. 68, 1999 (1982)

17. K. Suzuki, R. Okamoto, Prog. Theor. Phys. 92, 1045 (1994)

18. A.C. Hayes, P. Navrátil, J.P. Vary, Phys. Rev. Lett. 91, 012502 (2003)

19. E. Caurier, P. Navrátil, Phys. Rev. C 73, 021302 (2006) 
20. A. Nogga, P. Navratil, B.R. Barrett, J.P. Vary, Phys. Rev. C 73, 064002 (2006)

21. P. P. Navrátil, V.G. Gueorguiev, J.P. Vary, W.E. Ormand, A. Nogga, Phys. Rev. Lett. 99, 042501 (2007)

22. S. Quaglioni, P. Navrátil, Phys. Lett. B652, 370 (2007)

23. I. Stetcu, C.P. Liu, J.L. Friar, A.C. Hayes, P. Navratil, Phys. Lett. B665, 168 (2008)

24. I. Stetcu, B.R. Barrett, P. Navrátil, J.P. Vary, Phys. Rev. C 71, 044325 (2005)

25. P.F. Bedaque, U. van Kolck, Ann. Rev. Nucl. Part. Sci. 52, 339 (2002)

26. U. van Kolck, Nucleon nucleon interaction and isospin violation, in Workshop on Chiral Dynamics 1997, Theory and Experiment, edited by A. Bernstein, D. Drechsel, T. Walcher (Springer-Verlag, 1997), hep-ph/9711222

27. U. van Kolck, Nucl. Phys. A645, 273 (1999)

28. M. Köhl, H. Moritz, T. Stöferle, K. Günter, T. Esslinger, Phys. Rev. Lett. 94, 080403 (2005)

29. T. Stöferle, H. Moritz, K. Günter, M. Köhl, T. Esslinger, Phys. Rev. Lett. 96(3), 030401 (2006)

30. T. Busch, B.G. Englert, K. Rza̧żewski, M. Wilkens, Found. Phys. 28, 549 (1998)

31. I. Stetcu, J. Rotureau, B.R. Barrett, U. van Kolck (2009), to be submitted

32. A. Bhattacharyya, T. Papenbrock, Phys. Rev. A 74, 041602(R) (2006)

33. T. Mehen, Physical Review A 78(1), 013614 (2008)

34. F. Werner, Y. Castin, Phys. Rev. Lett. 97, 150401 (2006)

35. F. Werner, Y. Castin, Phys. Rev. A 74, 053604 (2006)

36. Y. Alhassid, G.F. Bertsch, L. Fang, Phys. Rev. Lett. 100, 230401 (2008)

37. P.F. Bedaque, H.W. Hammer, U. van Kolck, Phys. Rev. C 58, R641 (1998)

38. P.F. Bedaque, H.W. Hammer, U. van Kolck, Phys. Rev. Lett. 82, 463 (1999)

39. P.F. Bedaque, H.W. Hammer, U. van Kolck, Nucl. Phys. A646, 444 (1999)

40. P.F. Bedaque, H.W. Hammer, U. van Kolck, Nucl. Phys. A676, 357 (2000)

41. P.F. Bedaque, E. Braaten, H.W. Hammer, Phys. Rev. Lett. 85, 908 (2000)

42. J. Rotureau, I. Stetcu, B.R. Barrett, M. Birse, U. van Kolck (2010), to be submitted 